\title{
Gamma Heavy Chain Disease
}

National Cancer Institute

\section{Source}

National Cancer Institute. Gamma Heavy Chain Disease. NCI Thesaurus. Code C3083.

A clonal disorder characterized by the secretion of a truncated gamma chain. In most cases, it is associated with morphologic changes also seen in lymphoplasmacytic lymphomas, but the clinical course is typically more aggressive than in lymphoplasmacytic lymphoma. 